\title{
Adult Primary Prostate Embryonal Rhabdomyosarcoma: Report of a Case and Revue of Literature
}

\author{
Lamiae Amaadour ${ }^{1}$, Yassir Tahiri' ${ }^{2}$, Hassania Ameurtesse ${ }^{3}$, Moustapha Ahssaini'2, \\ Zineb Benbrahim ${ }^{1}$, Taoufik Harmouche ${ }^{3}$, Samia Arifi ${ }^{1}$, Jalaleddine El Ammari², \\ Nawfel Mellas ${ }^{1}$ \\ ${ }^{1}$ Department of Medical Oncology, Hassan II University Hospital, Fez, Morocco \\ ${ }^{2}$ Department of Urology, Hassan II University Hospital, Fez, Morocco \\ ${ }^{3}$ Department of Pathology, Hassan II University Hospital, Fez, Morocco \\ Email: lamiae.amaadour@gmail.com
}

Received 19 April 2014; revised 12 May 2014; accepted 19 May 2014

Copyright @ 2014 by authors and Scientific Research Publishing Inc.

This work is licensed under the Creative Commons Attribution International License (CC BY). http://creativecommons.org/licenses/by/4.0/

c) (i) Open Access

\begin{abstract}
Introduction: Primary embryonal rhabdomyosarcoma of prostate (ERMP) is an extremely rare pathological and clinical entity in adults. It's characterized by extensive loco-regional spread and a tendency to metastasize by blood stream and regional lymphatics. In adult patients, data available on the management of ERMP are limited and mostly derived from the pediatric clinical trials on rhabdomyosarcoma. Case Report: We report a case of group III ERMP approached by initial surgery followed by the administration of three courses of vincristine, doxorubicin and Cyclophosphamide regimen. The patient succumbed to disease complications three months after the original diagnosis. Conclusion: The most appropriate therapeutic approach for adults with ERMP remains questionable. Further studies are needed to improve the understanding of biological behavior of ERMP in adults and to define the best therapeutic strategy in locally advanced disease.
\end{abstract}

\section{Keywords}

Prostate Rhabdomyosarcoma, Multimodal Therapy, Adult

\section{Background}

Prostate rhabdomyosarcoma is a highly malignant tumor predominantly found in children. In adults, this tumor is extremely rare and clinical experience is limited to case reports. The present study reports a case of an adult patient 
with embryonal rhabdomyosarcoma of the prostate and comprehensively reviews the world literature on this subject.

\section{Case Report}

A 38 years old man presented with two months history of increased frequency of urination and dysuria followed by acute urinary obstruction. On examination, he had grade 2 painefulprostatomegaly with no lymphadenopathy or hepatosplenomegaly. Blood counts, urine microscopy, renal and liver function tests were normal. Prostate specific antigen (PSA) level was $4.54 \mathrm{ng} / \mathrm{ml}$. A magnetic resonance imaging (MRI) of the abdomen and pelvis revealed anheterogenous mass rising from the prostate and involving seminal vesicals (Figure 1). There was no evidence of lymph adenopathy or liver metastasis. The patient underwent transurethral resection and histology supplemented by immunochemistry showed sheets of spindle cells having a moderate amount of eosinophilic cytoplasm and hyperchromatic nuclei (Figure 2), expressing desmin and myogenin (Figure 3) and negative for cytokeratin, suggestive of embryonal RMS Chest computed tomographyand bone scan were normal.

The patient underwent initial radical cystoprostatectomy with residual gross macroscopic tumor; the reason of incomplete resection was pelvic shielding. Three weeks after surgery, the patient was started on vincristine (2 $\mathrm{mg})$, doxorubicin $\left(50 \mathrm{mg} / \mathrm{m}^{2}\right)$ and cyclophosphamide $\left(1500 \mathrm{mg} / \mathrm{m}^{2}\right)$ regimen every three weeks. After three courses of chemotherapy, the patient experienced a transient initial clinical response and radiological stability.
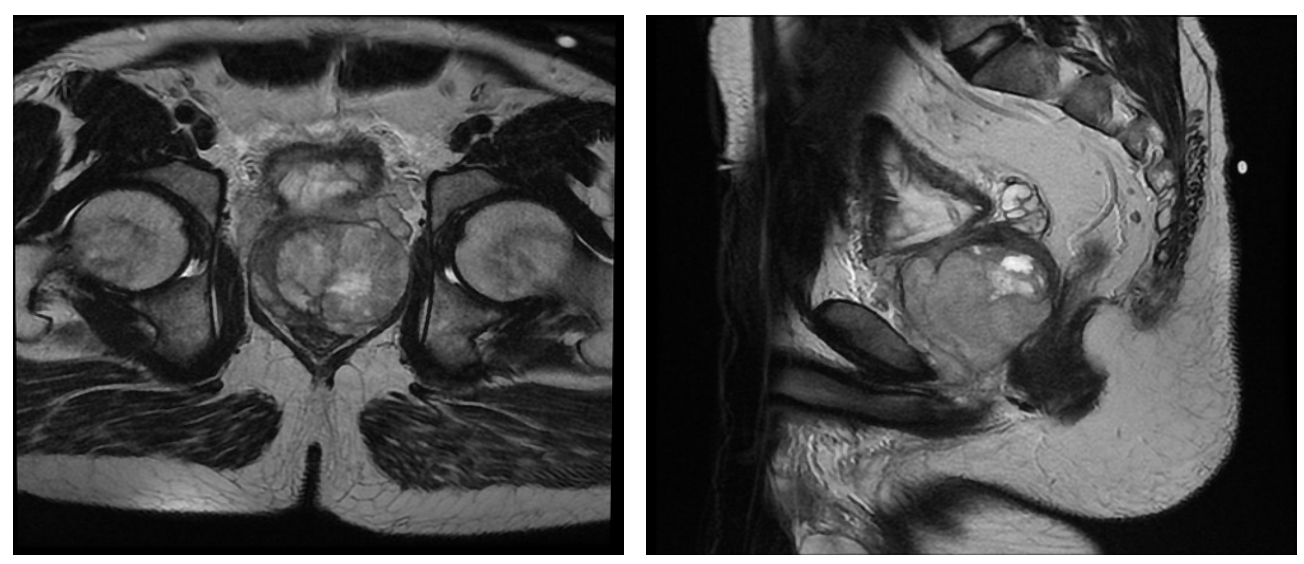

Figure 1. MRI of the pelvis showing expansive prostatic growth with invasion of seminal vesicals.

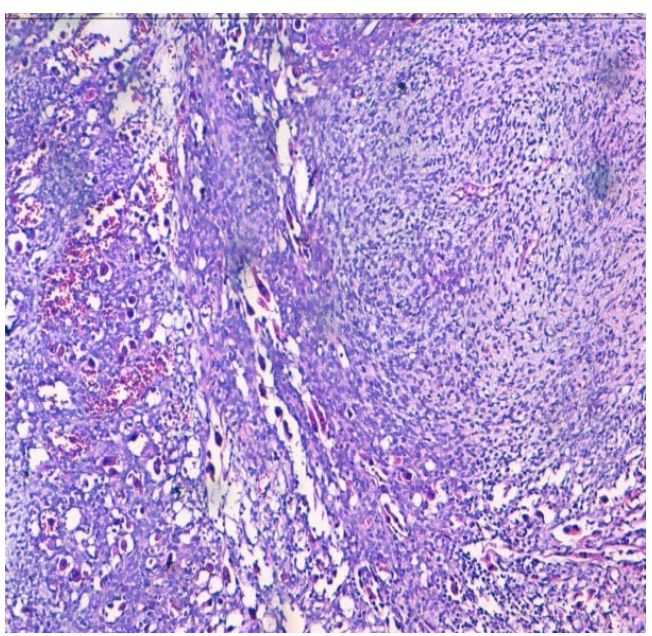

(a)

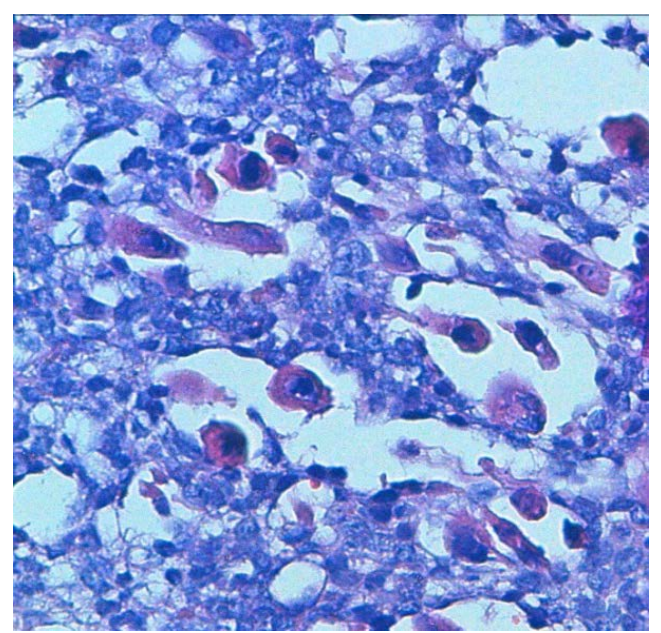

(b)

Figure 2. (a) HES stained image showing sheets of spindle cells containing round rhabdomyoblasts with eosinophilic cytoplasm and multiple hyperchromatic nuclei, at 20× magnification. (b) HES stained image showing rhabdomyoblasts at $40 \times$. 


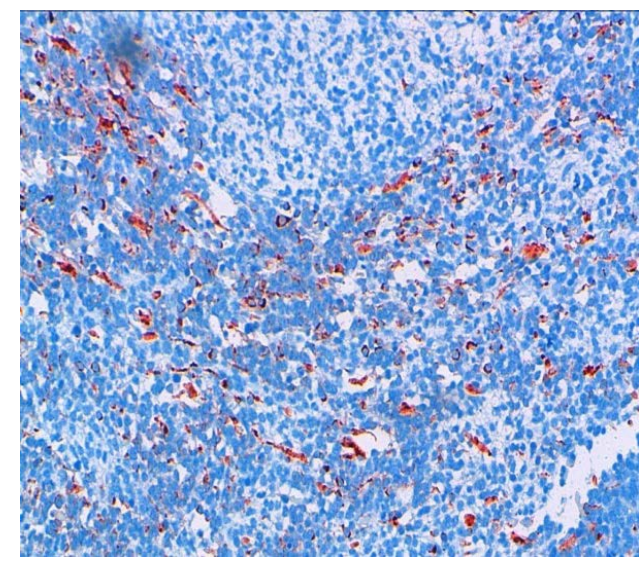

(a)

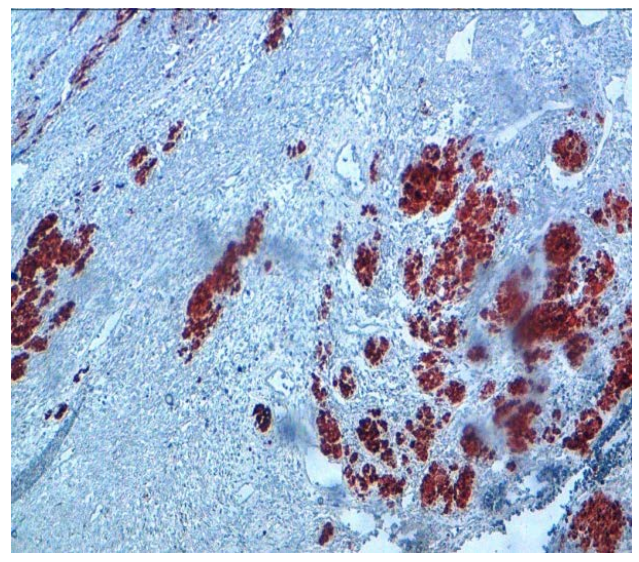

(b)

Figure 3. Immunochemistry $20 \times$ image showing desmin positivity (a) and myogenin positivity (b).

He succumbed to complications of widespread disease three months after the original diagnosis.

\section{Discussion}

Embryonal rhabdomyosarcoma of prostate (ERMP) is a common tumor in infants and children, with a median occurrence age of 5 years, but it is rare in adults [1] [2]. It is characterized by a high degree of malignancy, both local rapid growth with formation of large pelvic masses, often leading to renal failure due to urethral obstruction, and systemic spread, commonly to the lungs, liver bone and regional lymph nodes [3]. The histogenesis of embryonal rhabdomyosarcomas is curious because they have a propensity to arise in tissues, such as the middle ear, bile duct, bladder, and prostate, that are devoid of skeletal muscle. They are thought to derive from undifferentiated mesenchymal cells, which in the distal urogenital tract surround the mesonephric duct and become incorporated into the bladder and prostate during embryogenes [4]. These cells, which persist into later life, have the capacity for rhabdomyoblastic differentiation, and may explain the occurrence of these tumors, albeit extremely rarely, in adults. The presenting symptoms of ERMP published in literature are often related to urethral obstruction. Radiologic evaluation of genitourinary organs and potential intra-abdominal metastatic sites can be accomplished with computed tomography (CT) of the abdomen and pelvis [5] [6]. Recently, the improved contrast resolution of magnetic resonance imaging (MRI) has offered new potential for staging primary tumors. In fact, the use of longitudinal T1-weighted images in MRI reduces false impressions of tumor spread by reducing the partial-volume averaging effect encountered in CT scanning [5]. However, the radiological differential diagnosis with prostate adenocarcinoma can be very difficult. Chest CT is used to detect lung metastases. Bone scan, bone marrow aspirate and biopsy complete the metastatic work up [7]. The utilization of PET-CT for ERMP staging in adults is still to be consolidated in the literature, however, some reports have demonstrated the relevance of this method in the detection of the primary focus in metastatic disease or obscure metastases and the persistent viable disease or recurrence in a previously operated field, which is difficult to assess with current imaging methods [8] [9]. The diagnosis is made on transrectal needle biopsy or transurethral resection or biopsy specimens [10]. On histologic examination, embryonal rhabdomyosarcoma have high cytologic variability, which represents several stages of skeletal muscle morphogenesis. They may range from highly differentiated neoplasms containing rhabdomyoblasts with large amounts of eosinophilic cytoplasm and cross striations similar to that of poorly differentiated tumor cells. Desmin and muscle specific actin are the typical stains used to identify rhabdomyosarcoma [11]; newer staining agents, such as myogenin and MyoD1, are more specific for skeletal muscle than older stains. ERMS have unique molecular characteristic which consist of loss of the material from the 11p15 region [12].

The prognosis of adults individuals with rhabdomyosarcoma (RMS) remains poor. Recently, Ferrari and al [13] reported in a large retrospective analysis of 141 adults with rhabdomyosarcoma, thirty six genitourinary RMS submitted to multimodal treatment according to guidelines childhood RMS. In this report, the 5-year event-free survival and 5-years overall survival were 32.9 months and 45.7 months respectively, suggesting the worse outcome of adults with RMS than children; however, subgroup analysis showed that genitourinary RMS 
were not associated with the worst reported outcomes. Other previous series of adults prostatic rhabdomyosarcoma have reported poor results and significantly different natural history of genitourinary tract tumors in these patients in comparison to children [14]-[16].

Multimodal therapy is essential to cure patients with RMS, but different uses of surgery, radiotherapy and chemotherapy, and their intensity, need to be selected and modulated to different patient risk groups. In adult patients, data available on the management of RMS are limited and mostly from heterogeneous retrospective single institution studies. Therapeutic options used are usually derived from the pediatric clinical trials on RMS conducted by international cooperative groups, but no definitive conclusions could be drawn up to now regarding the best available treatment option towards ERMP in adults.

The treatment policy largely depends on the disease stage at the time of appearance. Localized prostatic RMS require prostatectomy with an attempt at bladder salvage with or without urethral reconstruction [17]. Unfortunately, the functional morbidity of initial surgery for genitourinary tract tumors has prompted the avoidance of upfront surgery in favor of multimodality approaches [18]. Prostatic RMS usually respond well enough to induction chemotherapy to render them locally resectable, often with clear resection margins [19] [20]. For patients with group III (clinical group classification [21] [22]) embryonal disease as in our patient's case, VAC chemotherapy (vincristine, dactinomycin, cyclophosphamide) or VAC alterning with vincristine and irinotecan [23] [24] is the standard treatment. The addition of many individually active agents (like doxorubicin, cisplatin, etoposide, ifosfamide, topotecan, and melphalan) did not improve outcomes, compared to VAC [25]-[27]. Radiotherapy is a major tool for achieving local control after chemotherapy or surgery for group III patients. The optimal timing and sequence of RT is not established; it is generally started at week 4 of therapy and given at the doses of 45 to 50.4 gray [28] [29]. The resection of post therapy residual tumor (second look surgery) has been evaluated in group III patients for the purpose of resecting viable tumor that remains after chemotherapy and radiotherapy in the hope of improving outcomes. This approach was beneficial for confirming the pathologic tumor response, for identifying patients who required second-line chemotherapy. However, the impact of a second-look procedure on survival in group III patients was unclear [21] [30]. In general, modern risk-based multimodality therapeutic protocols including chemotherapy for primary cytoreduction and eradication of both macroscopic and microscopic disease, surgery if feasible, and radiation therapy to control microscopic local residual disease, leads to improved outcomes in children with RMS [23]. However, regimens specifically designed for children may not be the most suitable ones for adults; who may be able to tolerate more intense regimens than those used to treat children, but not necessarily those developed specifically for children. So in the absence of large experience with treatment of adults with ERMP, those ones should be treated using the same principles as established for children, especially astherapeutic results are most favorable when adults are treated in pediatric clinical trials, which are generally open to individuals up to age 50 [13] [31] [32], and although data are lacking with regards to some specifics such as the total radiation dose and the best way to integrate surgery, radiation, and chemotherapy in these patients.

\section{Conclusion}

More data and further studies are needed in the future in order to improve the understanding of biological behavior of ERMP in adults and to define the most appropriate therapeutic approach, as well as the timing of local therapy in locally advanced disease.

\section{Disclosure}

All authors declare no conflict of interest.

\section{References}

[1] Nabi, G., Dinda, A.K. and Dogra, P.N. (2002) Primary Embryonal Rhabdomyosarcoma of Prostate in Adults: Diagnosis and Management. International Urology and Nephrology, 34, 531-534. http://dx.doi.org/10.1023/A:1025638711476

[2] Figueiredo, A.A., Brandão, G., Dentz, L.C., Alves, C.M. and Stroppa, A.E. (2007) Bilateral Pulmonary Alveolar Infiltrate and Prostate Tumour in a 54-Year-Old Man. Thorax, 62, 1002-1018. http://dx.doi.org/10.1136/thx.2007.083717

[3] Waring, P.M. and Newland, R.C. (1992) Prostatic Embryonal Rhabdomyosarcoma in Adults. A Clinicopathologic Review. Cancer, 69, 755-762. 
[4] Batsakis, J.G. (1963) Urogenital Rhabdomyosarcoma: Histiogenesis and Classification. Journal of Urology, 90, 180186.

[5] Kaefer, M. and Rink, R.C. (2000) Genitourinary Rhabdomyosarcoma. Treatment Options. Urologic Clinic of North America, 27, 471-487. http://dx.doi.org/10.1016/S0094-0143(05)70095-1

[6] Ciammella, P., Galeandro, M., D’Abbiero, N., Palmieri, T., Donini, E. and Iotti, C. (2013) Prostate Embryonal Rhabdomyosarcoma in Adults: Case Report and Review of Literature. Reports of Practical Oncology and Radiotherapy, 1, 8310-8315.

[7] Wu, H.-Y. (2009) Genitourinary Rhabdomyosarcoma: Which Treatment, How Much, and When? Journal of Pediatric Urology, 5, 501e-506. http://dx.doi.org/10.1016/j.jpurol.2009.06.011

[8] Klem, M.L., Grewal, R.K., Wexler, L.H., Schöder, H., Meyers, P.A. and Wolden, S.L. (2007) PET for Staging in Rhabdomyosarcoma: An Evaluation of PET as an Adjunct to Current Staging Tools. Journal of Pediatric Hematology/Oncology, 29, 9-14. http://dx.doi.org/10.1097/MPH.0b013e3180307693

[9] Kleis, M., Daldrup-Link, H., Matthay, K., Goldsby, R., Lu, Y., Schuster, T., Schreck, C., Chu, P.W., Hawkins, R.A. and Franc, B.L. (2009) Diagnostic Value of PET/CT for the Staging and Restaging of Pediatric Tumors. European Journal of Nuclear Medicine and Molecular Imaging, 36, 23-36. http://dx.doi.org/10.1007/s00259-008-0911-1

[10] Bisceglia, M., Magro, G., Carosi, I., Cannazza, V. and Ben Dor, D. (2011) Primary Embryonal Rhabdomyosarcoma of the Prostate in Adults: Report of a Case and Review of the Literature. International Journal of Surgical Pathology, 19, 831-837. http://dx.doi.org/10.1177/1066896910395585

[11] Drake, A.F., Lee, S.C. and Kelley, D.J. (2012) Pathology—Rhabdomyosarcoma. Medscape. http://emedicine.medscape.com/article/873546-overview\#aw2aab6b3

[12] Barr, F.G. (1997) Molecular Genetics and Pathogenesis of Rhabdomyosarcoma. Journal of Pediatric Hematology/Oncology, 19, 483-491. http://dx.doi.org/10.1097/00043426-199711000-00001

[13] Ferrari, A., Dileo, P., Casanova, M., Bertulli, R., Meazza, C., Gandola, L., Navarria, P., Collini, P., Gronchi, A., Olmi, P., Fossati-Bellani, F. and Casali, P.G. (2003) Rhabdomyosarcoma in Adults. A Retrospective Analysis of 171 Patients Treated at a Single Institution. Cancer, 98, 571-580. http://dx.doi.org/10.1002/cncr.11550

[14] Waring, P.M. and Newland, R.C. (1992) Prostatic Embryonal Rhabdomyosarcoma in Adults. A Clinicopathologic Review. Cancer, 69, 755-762. http://dx.doi.org/10.1002/1097-0142(19920201)69:3<755::AID-CNCR2820690324>3.0.CO;2-Y

[15] Esnaola, N.F., Rubin, B.P., Baldini, E.H., et al. (2001) Response to Chemotherapy and Predictors of Survival in Adult Rhabdomyosarcoma. Annals of Surgery, 234, 215-223. http://dx.doi.org/10.1097/00000658-200108000-00012

[16] Russo, P. (1991) Urologic Sarcoma in Adults: Memorial Sloan-Kattering Cancer Center Experience Based on a Prospective Database between 1982 and 1989. Urologic Clinics of North America, 18, 581-588.

[17] Hays, D.M., Lawrence Jr., W., Crist, W.M., Wiener, E., Raney Jr., R.B., Ragab, A., Teffr, M., Webber, B., Johnston, J., Maurer, H.M. and Intergroup Rhabdomyosarcoma Study (1990) Partial Cystectomy in the Management of Rhabdomyosarcoma of the Bladder: A Report from the Intergroup Rhabdomyosarcoma Study. Journal of Pediatric Surgery, 25, 719-723. http://dx.doi.org/10.1016/S0022-3468(05)80004-1

[18] Michalkiewicz, E.L., Rao, B.N., Gross, E., et al. (1997) Complications of Pelvic Exenteration in Children Who Have Genitourinary Rhabdomyosarcoma. Journal of Pediatric Surgery, 32, 1277-1282. http://dx.doi.org/10.1016/S0022-3468(97)90301-8

[19] Martelli, H., Oberlin, O., Rey, A., Godzinski, J., Spicer, R.D., Bouvet, N., Haie-Meder, C., Terrier-Lacombe, M.J., Sanchez de Toledo, J., Spooner, D., Sommelet, D., Flamant, F. and Stevens, M.C. (1999) Conservative Treatment for Girls with Nonmetastatic Rhabdomyosarcoma of the Genital Tract: A Report from the Study Committee of the International Society of Pediatric Oncology. Journal of Clinical Oncology, 17, 2117-2122.

[20] Heyn, R., Newton, W.A., Raney, R.B., Hamoudi, A., Bagwell, C., Vietti, T., Wharam, M., Gehan, E. and Maurer, H.M. (1997) Preservation of the Bladder in Patients with Rhabdomyosarcoma. Journal of Clinical Oncology, 15, 69-75.

[21] Crist, W., Gehan, E.A., Ragab, A.H., et al. (1995) The Third Intergroup Rhabdomyosarcoma Study. Journal of Clinical Oncology, 13, 610.

[22] Crist, W.M., Garnsey, L., Beltangady, M.S., Gehan, E., Ruymann, F., Webber, B., Hays, D.M., Wharam, M. and Maurer, H.M. (1990) Prognosis in Children with Rhabdomyosarcoma: A Report of the Intergroup Rhabdomyosarcoma Studies I and II. Intergroup Rhabdomyosarcoma Committee. Journal of Clinical Oncology, 8, 443-452.

[23] Crist, W.M., Anderson, J.R., Meza, J.L., Fryer, C., Raney, R.B., Ruymann, F.B., Breneman, J., Qualman, S.J., Wiener, E., Wharam, M., Lobe, T., Webber, B., Maurer, H.M. and Donaldson, S.S. (2001) Intergroup Rhabdomyosarcoma Study-IV: Results for Patients with Nonmetastatic Disease. Journal of Clinical Oncology, 19, 3091-3102.

[24] (2011) Clinical Trial Information for ARST0531 (NCT00354835). 
http://cancer.gov/clinicaltrials/search/view?cdrid=487560\&version=healthprofessional

[25] Ferrer, F.A., Isakoff, M. and Koyle, M.A. (2006) Bladder/Prostate Rhabdomyosarcoma: Past, Present and Future. Journal of Urology, 176, 1283-1291. http://dx.doi.org/10.1016/j.juro.2006.06.019

[26] Little, D.J., Ballo, M.T., Zagars, G.K., Pisters, P.W.T., Patel, S.R., El-Naggar, A.K., Garden, A.S. and Benjamin, R.S. (2002) Adult Rhabdomyosarcoma: Outcome Following Multimodality Treatment. Cancer, 95, 377-388. http://dx.doi.org/10.1002/cncr.10669

[27] Arndt, C.A., Stoner, J.A., Hawkins, D.S., Rodeberg, D.A., Hayes-Jordan, A.A., Paidas, C.N., Parham, D.M., Teot, L.A., Wharam, M.D., Breneman, J.C., Donaldson, S.S., Anderson, J.R. and Meyer, W.H. (2009) Vincristine, Actinomycin, and Cyclophosphamide Compared with Vincristine, Actinomycin, and Cyclophosphamide Alternating with Vincristine, Topotecan, and Cyclophosphamide for Intermediate-Risk Rhabdomyosarcoma: Children's Oncology Group Study D9803. Journal of Clinical Oncology, 27, 5182-5188. http://dx.doi.org/10.1200/JCO.2009.22.3768

[28] Wharam, M.D., Meza, J., Anderson, J., Breneman, J.C., Donaldson, S.S., Fitzgerald, T.J., Michalski, J., Teot, L.A., Wiener, E.S. and Meyer, W.H. (2004) Failure Pattern and Factors Predictive of Local Failure in Rhabdomyosarcoma: A Report of Group III Patients on the Third Intergroup Rhabdomyosarcoma Study. Journal of Clinical Oncology, 22, 1902-1908. http://dx.doi.org/10.1200/JCO.2004.08.124

[29] Okcu, F., Hicks, J. and Horowitz, M. (2014) Rhabdomyosarcoma and Undifferentiated Sarcoma in Childhood and Adolescence: Treatment. UPTODATE.

[30] Rodeberg, D.A., Stoner, J.A., Hayes-Jordan, A., Kao, S.C., Wolden, S.L., Qualman, S.J., Meyer, W.H. and Hawkins, D.S. (2009) Prognostic Significance of Tumor Response at the End of Therapy in Group III Rhabdomyosarcoma: A Report from the Children's Oncology Group. Journal of Clinical Oncology, 27, 3705-3711. http://dx.doi.org/10.1200/JCO.2008.19.5933

[31] Godzinski, J., Flamant, F., Rey, A., Praquin, M.T. and Martelli, H. (1994) Value of Postchemotherapy Bioptical Verification of Complete Clinical Remission in Previously Incompletely Resected (Stage I and II pT3) Malignant Mesenchymal Tumors in Children: International Society of Pediatric Oncology 1984 Malignant Mesenchymal Tumors Study. Medical and Pediatric Oncology, 22, 22-26. http://dx.doi.org/10.1002/mpo.2950220105

[32] Gerber, N.K., Wexler, L.H., Singer, S., Alektiar, K.M., Keohan, M.L., Shi, W.J., Zhang, Z.G. and Wolden, S. (2013) Adult Rhabdomyosarcoma Survival Improved with Treatment on Multimodality Protocols. International Journal of Radiation Oncology * Biology * Physics, 86, 58-63. http://dx.doi.org/10.1016/j.ijrobp.2012.12.016 